\title{
Sublingual Piroxicam as Preemptive Analgesia in Single Implant Surgery
}

\author{
Neha Bhutani ${ }^{1}$, Deepak Sangolikar ${ }^{2}$, Saurabh Bhutani ${ }^{3}$, Roopali Tapashetti ${ }^{4}$, Halmath Pushpalatha ${ }^{5}$
}

\begin{abstract}
Aim: The aim of this study is to evaluate the efficacy of sublingual piroxicam as a preemptive analgesic agent in patients undergoing single implant surgery.

Materials and methods: This was a prospective triple-blind placebo-controlled trial that involved 40 patients presented for the placement of a single endosteal implant in the posterior maxillary arch. Patients were randomly allocated to one of the following groups. Group I (study) who received sublingual piroxicam $40 \mathrm{mg} 1$ hour prior to the surgical procedure and group II (control) received placebo 1 hour before the surgery, both the groups received sublingual piroxicam $20 \mathrm{mg}$ tablet twice a day on the first and the second postoperative day and once a day on the postoperative day 3. Visual analog scores for measuring pain intensity were assessed at postoperative 1 hour, 6 hours, days 1, 3, and 5. Facial swelling was measured and evaluated during the first, third, and fifth postoperative days using the digital vernier caliper. The Mann-Whitney $U$ test was used to make between-group comparisons. The Wilcoxon-signed rank test was used to make within-group comparisons.

Results: At all-time intervals, both mean pain and mean swelling scores were higher in controls as compared with those of cases. The difference between the two groups was also statistically significant at all-time intervals for both parameters except for pain at 1 hour.

Conclusion: Our findings suggest that preoperative sublingual piroxicam is more effective for controlling postoperative pain and swelling after surgical implant placement than only postoperative administration.

Keywords: Preemptive analgesia, Single implant, Sublingual piroxicam.

The Journal of Contemporary Dental Practice (2019): 10.5005/jp-journals-10024-2591
\end{abstract}

\section{INTRODUCTION}

Postoperative pain and swelling are potential side effects of oral surgical procedures including the placement of dental implants. ${ }^{1}$ Despite the predictable success of dental implants, postoperative pain and swelling reduce the compliance of patients toward implant therapy. Hence, attempts should be made to prevent these complications that accompany tissue injury after implant placement to improve the quality of life of patient. ${ }^{2}$

The concept of preemptive analgesia involves the administration of analgesic before a painful stimulus is initiated. It consists of antinociceptive treatment that prevents central neural sensitization which amplifies postoperative pain. ${ }^{3}$

Nonsteroidal anti-inflammatory drugs (NSAIDs) have been used to obtain preemptive analgesia. NSAID inhibits cyclooxygenase enzyme and decreases peripheral and central prostaglandin production. This, in turn, reduces the response of peripheral and central sensitization to noxious stimuli. These properties make NSAID to be used as preemptive analgesia before a surgical procedure with an expectation of a reduction in the pain. ${ }^{4}$

Piroxicam is a NSAID with a long half-life ( 50 hours) and potent analgesic activity. ${ }^{5}$ The analgesic efficacy of sublingual piroxicam in several types of pain (e.g., postoperative, renal colic, osteoarthritis, lower back pain, and third molar surgery ${ }^{6}$ have been investigated) NO study has evaluated the prevention and control of pain and swelling following dental implant placement. The aim of our study is to evaluate the efficacy of sublingual piroxicam medication protocol for postoperative pain and swelling control following implant surgery.

\section{Materials and Methods}

This was a prospective, triple-blind, placebo-controlled randomized clinical trial that involved 40 patients in the age group of $16-40$ years
1Department of Periodontics, Al Badar Rural Dental College and Hospital, Kalaburagi, Karnataka, India

${ }^{2}$ Department of Oral and Maxillofacial Surgery, S B Patil Dental College and Hospital, Bidar, Karnataka, India

${ }^{3}$ Department of Pharmaceutics, HIMT College of Pharmacy, Greater Noida, Uttar Pradesh, India

${ }^{4} \mathrm{Al}$ Badar Rural Dental College and Hospital, Kalaburagi, Karnataka, India ${ }^{5}$ Department of Pedodontics, Al Badar Rural Dental College and Hospital, Kalaburagi, Karnataka, India

Corresponding Author: Neha Bhutani, Department of Periodontics, Al Badar Rural Dental College and Hospital, Kalaburagi, Karnataka, India, Phone: +91 9916807389, e-mail: nehabhutaniperio@gmail.com

How to cite this article: Bhutani N, Sangolikar D, et al. Sublingual Piroxicam as Preemptive Analgesia in Single Implant Surgery. JContemp Dent Pract 2019;20(6):750-753.

Source of support: Nil

Conflict of interest: None

who presented to the Department of Periodontics, Albadar Rural Dental College, Kalaburagi, for the placement of the single endosteal implant in the posterior maxillary arch irrespective of gender. All patients were explained about the study and informed consent was obtained from them. Ethical committee clearance was obtained before starting the study. Exclusion criteria included the history of allergic reaction to local anesthetics; history of pain, signs of infection or other related problems existing in the 3 weeks before the surgical procedure; allergy to aspirin, piroxicam, or any other NSAIDs.

Patients were randomly allocated to one of the following groups. Group I (study) who received sublingual piroxicam $40 \mathrm{mg}$ 1 hour prior to the surgical procedure and group II (control) received placebo 1 hour before the surgery, both the groups received

(0) The Author(s). 2019 Open Access This article is distributed under the terms of the Creative Commons Attribution 4.0 International License (https://creativecommons. org/licenses/by-nc/4.0/), which permits unrestricted use, distribution, and non-commercial reproduction in any medium, provided you give appropriate credit to the original author(s) and the source, provide a link to the Creative Commons license, and indicate if changes were made. The Creative Commons Public Domain Dedication waiver (http://creativecommons.org/publicdomain/zero/1.0/) applies to the data made available in this article, unless otherwise stated. 
sublingual piroxicam $20 \mathrm{mg}$ tablet twice a day on the first and second postoperative days and once a day on the postoperative day 3.

All procedures were performed by a single operator; the patients, operating surgeon, and the evaluating doctor were blinded throughout the study. All anesthetic and surgical protocol for implant placement was followed for each patient. All patients received amoxicillin $500 \mathrm{mg}$ thrice a day for 5 days.

All patients were assessed at postoperative 1 hour, 6 hours, days 1,3 , and 5 for pain and postoperative days 1, 3, and 5 for swelling. The subjective pain evaluation was documented with a visual analog scale (VAS) of $10 \mathrm{~cm}$ using a ruler which was shown to the patient with 0 representing "no pain" and 10 representing "severe pain".

The swelling in each patient was measured using following measurements: (1) the distance between the lateral corner of the eye and the angle of the mandible; (2) the distance between the tragus of the ear and the outer corner of the mouth. The preoperative sum of these two measurements was considered as the base value. The measurements were also recorded on the first third and fifth postoperative days. The difference between the measurement values and base values indicated the facial swelling for that day and graded as 0 ("no swelling", $<10 \mathrm{~mm}$ ), grade I ("mild swelling," 10-20 mm), grade II ("moderate swelling," 20-30 mm), and grade III ("severe swelling," >30 mm).

\section{Result}

In the postoperative period, at all-time intervals except for the pain at 1 hour, both mean pain and mean swelling scores were higher in controls as compared with those of the study group. The difference between two groups was also statistically significant $(p<0.05)$ (Table 1 and Figs 1 and 2).

For swelling, between day 1 and the third day, a significant decrease was observed in the study group but not in the control group. However, between the third day and the fifth day, a significant reduction in swelling was observed in both the groups (Table 2).

\section{Discussion}

The present study evaluated the efficacy of sublingual piroxicam as preemptive analgesia in a patient undergoing single implant surgery and suggested that the medication protocol is effective for preventing pain and swelling following the surgical procedure. The results supported the hypothesis of preemptive administration of sublingual piroxicam 1 hour before the surgical procedure.

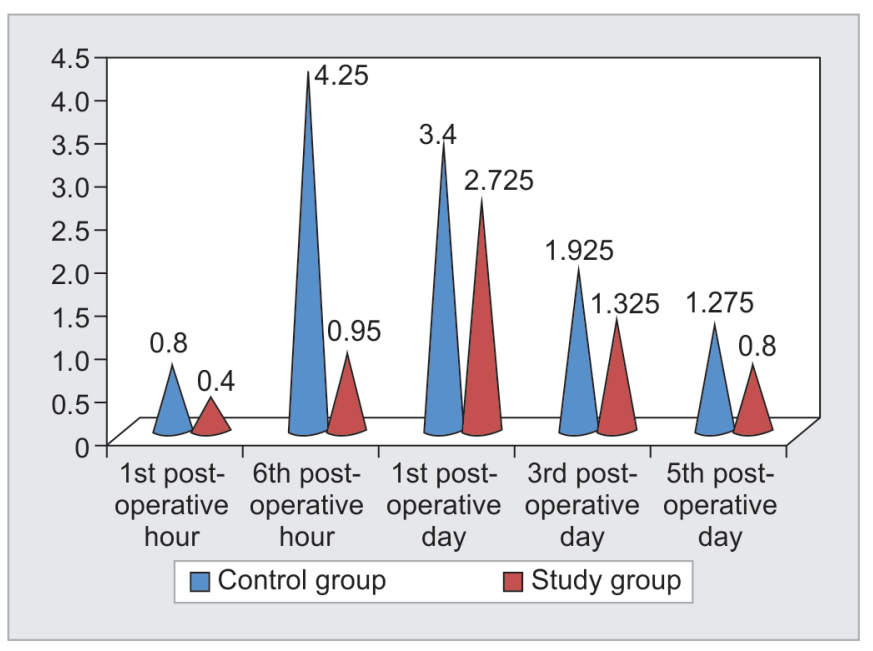

Fig. 1: Means of pain values

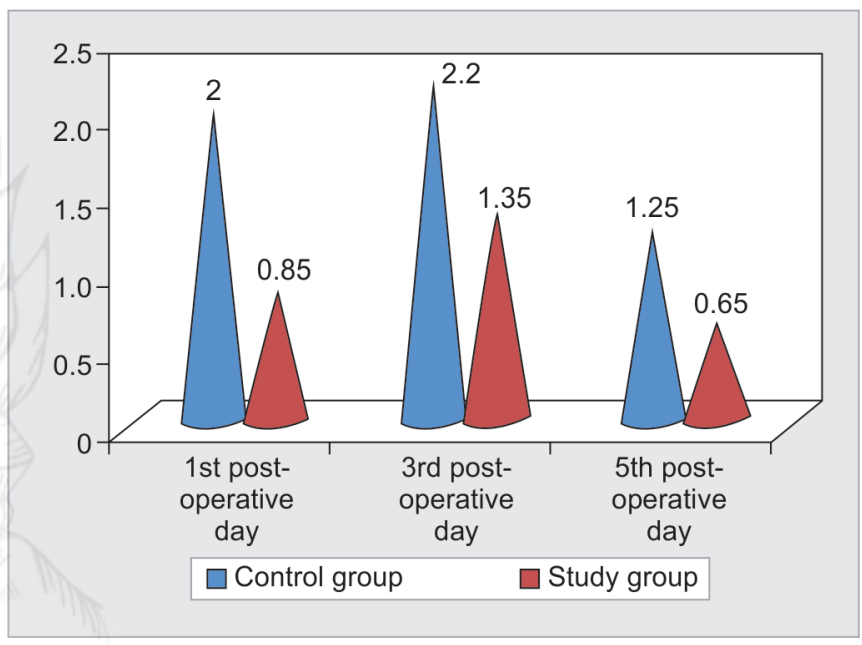

Fig. 2: Means of swelling values

Using the formula, $n=\left(Z^{2}(P)(1-P) / C^{2}\right.$, it was determined that 20 patients per group were needed in the study, considering the standard normal deviation set at 95\% confidence level (1.96) and percentage picking a choice or response $(80 \%=0.8)$. The technique used for randomization in the present study was the simple random sampling.

Table 1: Comparison between experimental and control groups using the Mann-Whitney $U$ test

\begin{tabular}{|c|c|c|c|c|c|}
\hline & \multicolumn{2}{|c|}{ Control group } & \multicolumn{2}{|c|}{ Study group } & \multirow[b]{2}{*}{$p$ value } \\
\hline & Mean & $S D$ & Mean & $S D$ & \\
\hline \multicolumn{6}{|l|}{ Pain values } \\
\hline 1st postoperative hour & 0.8 & 0.6782 & 0.4 & 0.6633 & 0.0767 (NS) \\
\hline 6th postoperative hour & 4.25 & 0.7665 & 0.95 & 0.2179 & $<0.00001(\mathrm{~S})$ \\
\hline 1st postoperative day & 3.4 & 0.5612 & 2.725 & 0.8437 & $0.0117(\mathrm{~S})$ \\
\hline 3rd postoperative day & 1.925 & 0.8258 & 1.325 & 0.6759 & $0.0434(\mathrm{~S})$ \\
\hline 5th postoperative day & 1.275 & 0.5356 & 0.8 & 0.5099 & $0.0168(S)$ \\
\hline \multicolumn{6}{|l|}{ Swelling values } \\
\hline 1st postoperative day & 2.0 & 0.7071 & 0.85 & 0.4769 & $<0.00001(\mathrm{~S})$ \\
\hline 3rd postoperative day & 2.2 & 0.5099 & 1.35 & 0.4769 & $0.0002(S)$ \\
\hline 5th postoperative day & 1.25 & 0.4330 & 0.65 & 0.4769 & $0.0058(S)$ \\
\hline
\end{tabular}


Table 2: Comparison between consecutive two follow-ups using Wilcoxon-signed-rank test

\begin{tabular}{|c|c|c|c|c|}
\hline & \multicolumn{2}{|c|}{ Control group } & \multicolumn{2}{|c|}{ Study group } \\
\hline & Zvalue & $p$ value & Zvalue & pvalue \\
\hline \multicolumn{5}{|l|}{ Pain values } \\
\hline 1 st postoperative hour and 6th postoperative hour & -3.9199 & $<0.0001(\mathrm{~S})$ & -2.4990 & $0.0124(S)$ \\
\hline 6 th postoperative hour and 1st postoperative day & -3.1284 & $0.0017(S)$ & -3.7236 & $0.0002(S)$ \\
\hline 1st postoperative day and 3rd postoperative day & -3.6620 & $0.0003(S)$ & -3.4557 & $0.0005(S)$ \\
\hline 3rd postoperative day and 5th postoperative day & -2.2752 & $0.0226(S)$ & -2.2749 & $0.0232(S)$ \\
\hline \multicolumn{5}{|l|}{ Swelling values } \\
\hline 1st postoperative day and 3rd postoperative day & -0.8037 & 0.4237 (NS) & -2.6656 & $0.0145(S)$ \\
\hline 3rd postoperative day and 5th postoperative day & -3.3752 & $0.0007(S)$ & -3.1798 & $0.0015(S)$ \\
\hline
\end{tabular}

This was achieved by the Coin toss method wherein heads were assigned for the control group while tails were for the study group.

The drug administration protocol was in accordance with the study done by Mohammad et al. ${ }^{7}$ for sublingual piroxicam in the management of postoperative pain after surgical removal of the impacted mandibular third molar. However, unlike their study, the present study also used $40 \mathrm{mg}$ of sublingual piroxicam as a preemptive medication to ensure that there was no pain whatsoever after single implant placement.

The pain intensity, VAS scores at baseline, was less than $1 \mathrm{~cm}$ in both the treatment groups. The data were presented as mean with the significance level of 0.05 . The Mann-Whitney $U$ test was used to compare the VAS scores with a $p$ value $<0.05$ to be considered as statistically significant. The pain following minor oral surgeries is usually most felt during the 6th-8th hour postoperatively. Thus, the prime objective of the present study was to assess the VAS scores for the evaluation of pain at 6 hour postoperatively. The present study revealed that the preemptive drug was highly effective in reducing the VAS score in the test group compared with the control group at the 6th hour postoperatively. Also, the results were highly statistically significant with a $p$ value less than 0.0001 . However, the mean pain scores increased again during the 1st postoperative day in the study group. This increased score could be due to the very low scores that are achieved at the 6th postoperative hour by the effect of preemptive administration of the drug.

Similar to the present study, Gramke et al. ${ }^{8}$ investigated the use of sublingually administered piroxicam for preemptive analgesia in patients scheduled for laparoscopic bilateral inguinal hernia repair and found significantly lower visual analogue scores in the study group at 6 and 20 hour postoperatively and suggested that preoperative administration of sublingual piroxicam is more effective than the postoperative administration. In addition, the author also found less cumulative opioid consumption in the study group patients treated with preemptive sublingual piroxicam. Shah et al. ${ }^{9}$ evaluated the analgesic efficacy of intramuscular ketorolac following the third molar surgery and concluded that prolonged duration of pain relief with intramuscular ketorolac signifies the existence of preemptive analgesia.

In the present study, we have chosen the sublingual route of administration of drug due to the advantages offered by this route. This route avoids the first-pass metabolism in the liver and also the drug gets absorbed by the veins in the floor of the mouth, leading directly to superior vena cava, leading to the faster distribution of the drug..$^{10}$ However, conflicting to our view, Trindade et al. ${ }^{11}$ compared the oral vs sublingual piroxicam in the postoperative pain management after lower third molar extraction and found no significant difference in between the two routes of drug administration in terms of pain management.

Quantitative measurements of facial swellings are often used in research and attempts were made to relate the magnitude and duration of swelling to various drug regimes..$^{12}$ In our study, the method used to measure clinical swelling was simple, noninvasive, and provided numerical data using the digital vernier calliper which had a benefit of being reproducible. ${ }^{13}$ However, no additional radiographic analysis like computed tomography was done to support the clinical findings and also we have neglected the facts that swelling is a physical change in volume of tissues and also the considering variables of tissue tension during the recording procedures. The cephalostat was used in our study to standardize head posing during the recording session. Pendersen and Moller have described that the sterophotogrametric technique and Holland's face bow are the only 3D swelling volume determination methods in the literature to measure facial swelling. ${ }^{14}$ Yip et al. utilized the $3 D$ range camera and the Rugle $D$ volumetric measurement software for the assessment of facial swelling and reported a systemic error of only $1.25 \% .^{15}$

\section{ConClusion}

Our findings suggest that preoperative sublingual piroxicam is more effective for controlling postoperative pain and swelling after surgical implant placement than only postoperative administration.

\section{Clinical Significance}

Preemptive analgesia is a treatment that is initiated before the surgical procedure to reduce sensitization. Owing to this "protective" effect on the nociceptive system, immediate postoperative pain may be reduced and the development of chronic pain may be prevented.

\section{References}

1. Gonzalez SH, Penarrocha DM, et al. Pain and inflammation in 41 patients following the placement of 131 dental implants. Med Oral Patol Oral Cir Bucal 2005;10(3):258-263.

2. Hashem AA, Claffey NM, et al. Pain and anxiety following the placement of dental implants. Int J Oral Maxillofac Implants 2006;21(6):943-950.

3. Woolf CJ, Chong MS. Preemptive analgesia - treating postoperative pain by preventing the establishment of central sensitization. Anesth Analg 1993;77:362-379. DOI: 10.1213/00000539-199308000-00026.

4. Ochroch EA, Mardini IA, et al. What is the role of NSAIDs in pre-emptive analgesia? Drugs 2003;63(24):2709-2723. DOI: 10.2165/00003495200363240-00002. 
5. Yamamoto M, Sugano T, et al. Double-blind comparison of piroxicam and indomethacin in the treatment of cervicobrachial syndrome and periarthritis scapulohumeralis (stiff shoulder). Eur J Rheumatol Inflamm 1983;6(3):266-273.

6. TrindadePA, Giglio FP, et al.Sublingual ketorolac and sublingual piroxicam are equally effective for postoperative pain, trismus, and swelling management in lower third molar removal. Oral Surg Oral Med Oral Pathol Oral Radiol 2012 Jul;114(1):27-34.DOI: 10.1016/j.tripleo.2011.05.027.

7. Mohammad S, Singh V, et al. Sublingual piroxicam in the management of postoperative pain after surgical removal of impacted mandibular third molar. Indian J Dent Res 2012;23(6):839-840. DOI: 10.4103/09709290.111282.

8. Gramke HF, Petry JJ, et al. Sublingual piroxicam for postoperative analgesia: preoperative versus postoperative administration: a randomized, double-blind study. Anesth Analg 2006;102(3):755-758. DOI: 10.1213/01.ane.0000197611.89464.98.

9. Shah AV, Arun KV, et al. Comparative evaluation of pre-emptive analgesic efficacy of intramuscular ketorolac versus tramadol following third molar surgery. J Maxillofac Oral Surg 2013;12(2): 197-202. DOI: 10.1007/s12663-012-0420-4.
10. Graziani F, Corsi L, et al. Clinical evaluation of piroxicam-fddf and azithromycin in the prevention of complications associated with impacted lower third molar extraction. Pharmacol Res 2005:52:485490. DOI: 10.1016/j.phrs.2005.07.009.

11. Trindade PA, Giglio FP, et al. Comparison of oral versus sublingual piroxicam during postoperative pain management after lower third molar extraction. Int J Oral Maxillofac Surg 2011;40:292. DOI: 10.1016/j. ijom.2010.10.026.

12. Emad A. A new two dimensional facial contour tracing method. Bagh College Dentistry 2010;22(4):62-66.

13. de Santana-Santos T, de Souza-Santos aA, et al. Prediction of postoperative facial swelling, pain and trismus following third molar surgery based on preoperative variables. Med Oral Patol Oral Cir Bucal 2013;18(1):65-70.

14. Pedersen A, Moller OM. Volumetric determination of extraoral swelling from stereophotographs. Int J Oral Surg 1985;14:229-234. DOI: 10.1016/s0300-9785(85)80033-8.

15. Yip $E$, Smith $A$, et al. Volumetric evaluation of facial swelling utilizing a 3-D range camera. Int J Oral Maxillofac Surg 2004;2:179-182. DOI: 10.1054/ijom.2003.0501. 